\title{
Delay based Traffic Distribution of Heavy Traffic on K- Paths to achieve the Load Balancing and to minimize the Mean System Delay in MANET
}

\author{
Sushil Chandra Dimri \\ Professor \\ Graphic Era University \\ Dehradun- India
}

\author{
Sushil Kumar Chamoli \\ Assistant Professor \\ Graphic Era University \\ Dehradun- India
}

\author{
Durgesh Pant \\ Professor \\ Uttarakhand Open University \\ Dehradun- India
}

\begin{abstract}
Splitting of traffic allows the establishment of many paths between a pair of source and destination node in mobile ad hoc network. Splitting of traffic is typically proposed in order to increase the reliability of data transmission, to minimize the mean system delay and to provide load balancing. In this paper, we design for each path a queuing delay based traffic distribution scheme and implement a k-path routing that allows a given source node to send the data to a given destination node in a MANET. This approach of traffic distribution can increase the reliability of network system, provides the load balancing and minimization of system delay. The simulation results reveal that splitting of traffic approach perform better than the shortest path routing in terms of load balancing, reliability of network and in a minimum mean delay for the whole network.
\end{abstract}

\section{General Terms}

Mobile ad Hoc Network

\section{Keywords}

Single shortest path, k-Path routing, Mobile Ad Hoc network, Network reliability, Mean system delay

\section{INTRODUCTION}

Mobile ad hoc network (MANET) consist of a collection of wireless mobile nodes which dynamically exchange data among themselves without the reliance on a fixed base station or a wired backbone network. MANET nodes are typically distinguished by their limited power, processing, and memory resources as well as high degree of mobility. In such networks, the wireless mobile nodes may dynamically enter the network as well as leave the network. Due to the limited transmission range of wireless network nodes, multiple hops are usually needed for a node to exchange information with any other node in the network. Thus routing is a crucial issue to the design of a MANET [1 and 2].

When a node needs a route to another node, it initiates a route discovery process to find a route. Nowadays, Multipath routing is also being taken into consideration. Multipath routing allows the establishment of multiple paths between a pair of source and destination node [3 and4]. It is typically proposed in order to increase the reliability of data transmission or to provide load balancing and has received more and more attentions. The node works as a source, which generates data (traffic), destination and the router. Each node has limited memory, processing power and battery energy. The transmission and receiving range of a node is limited so multiple hops are required to transmit the data to the other distant nodes, which makes routing a crucial issue in mobile ad hoc network. There are many factors associated with MANET which makes routing a difficult issue in this mobile environment [5 and 6].

The main characteristics of the MANET are:
a) Temporary topology which changes over time
b) Limitation on resources
c) Wireless transmission
d) Network partitions
e) Limited bandwidths
f) Multi functionality of a node, as source, destination router and rely transmitter

The main challenges of mobile environment are:
a) Packet loss due to transmission error and limited processing power of a node
b) Variable capacities of links
c) Limited communication bandwidth
d) Broad cast nature of communication
e) battery life limited
f) Node mobility
g) Wireless medium

\subsection{Route establishment and route maintenance:}

The process of establishing routes consists of finding multiple routes between a source node and the destination node [7, 8 and 9]. (See Figure 1.1).

A Route Discovery is initiated when the source node attempts to discover routes to the destination by flooding request packets (RREQs). A RREQ consists of the source and destination addresses, a route record that stores the sequence of hops through which it has been propagated and a sequence number to prevent loops. The RREQ propagates until it reaches the target host.

- In MANET a route between any two nodes is established sending a route request by the source node to the neighboring nodes, from where this request it reaches to the destination node if the routes exist.

- This mechanism discovers multiple routes in the network between source and destination node.

- The route request first to arrive is accepted by the target. The target then responds on that route and intimates the initiator what the source routes are.

- In this way routes are established and then used to send the data traffic. 


\subsubsection{Maintenance of routes:}

This process in ad hoc network is used to repair the broken routes or finding alternative routes in case of routes failure.

- When the links lies on the alive routes between source and destination breaks then the existing routes does not work.

- When a node detects a broken link while attempting to forward a packet to the next hop, it generates an error message that is sent to all the sources using the broken link. If a source receive an error message and route to the destination is still required, it initiates a new route discovery process. Routes are also deleted from the routing table if they are unused for a certain amount of time.

- With help of route discovery mechanism an alternative route has established.

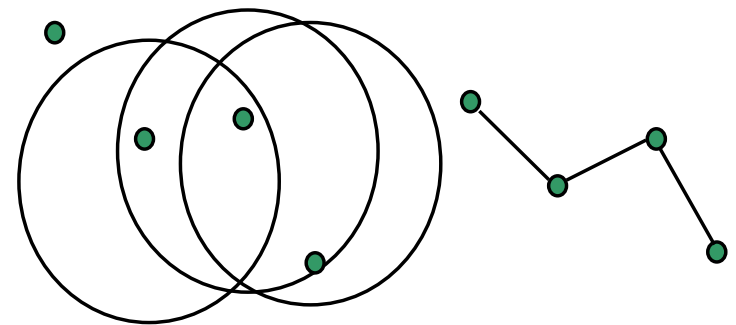

Fig1.1: The MANET and establishment of routes in MANET

Disjoint routes offer certain advantages over non-disjoint routes. For instance, non-disjoint routes may have lower aggregate resources than disjoint routes, because non-disjoint routes share links or nodes. In principle, node disjoint routes offer the most aggregate resources, because neither links nor nodes are shared between the paths. Disjoint routes also provide higher fault-tolerance. When using non-disjoint routes, a single link or node failure can cause multiple routes to fail. In node or link disjoint routes, a link failure will only cause a single route to fail. However, with link disjoint routes, a node failure can cause multiple routes that share that node to fail. Thus, node disjoint routes offer the highest degree of fault-tolerance [10 and 11].

\section{Single Path Routing}

In MANET single path routing is not an effective routing technique specially when there are many constraints. Single path routing sends entire traffic via a single route from source to destination. In MANET, the link capacity (bandwidth), the memory and processing power of the nodes are limited so that they cannot handle high amount of traffic, which generally leads to congestion ,packet loss, formation of hot spots in the network , as a consequences the end to end delay and unreliability of the network increases. In single path routing, if a link breaks or a node fails it leads to the network failure, i.e. no transmission occurs between source $\mathrm{S}$ and destination $\mathrm{T}$.

\section{Splitting of traffic to k-routes in MANET}

Splitting the traffic to different routes can provide better load balancing, fault tolerance and higher aggregate bandwidth. Splitting of the traffic can be helpful in reduction of congestion, bottle necks and to minimize the mean system delay, this also improves network resource utilization and bandwidth optimization [12 and 13].

\subsection{Network model for $k$-splittable routing:}

The single-path model is considered as a multi-node $\mathrm{M} / \mathrm{M} / 1$ tandem network, and the k-path model as a set of k- parallel path. The proposed framework allows us to investigate issues such as optimal load distribution, end-to-end delay and k- path routing reliability in ad hoc networks.

There are k node disjointed paths, which forms a sub graph in existing network, this sub graph is approximately a rectangular region, its size depend on source destination separation and the node density.

To model each multi-hop path, a multi node M/M/1 tandem network is considered [14, 15 and 16].

Assuming a path $\mathrm{p}(\mathrm{p}=1,2-\mathrm{-k})$ consists of $\mathrm{I}_{\mathrm{p}}$ intermediate relaying node .Then the $\mathrm{p}^{\text {th }}$ path can be modeled as a network in which $\mathrm{I}_{\mathrm{p}}$ queues are connected in tandem.

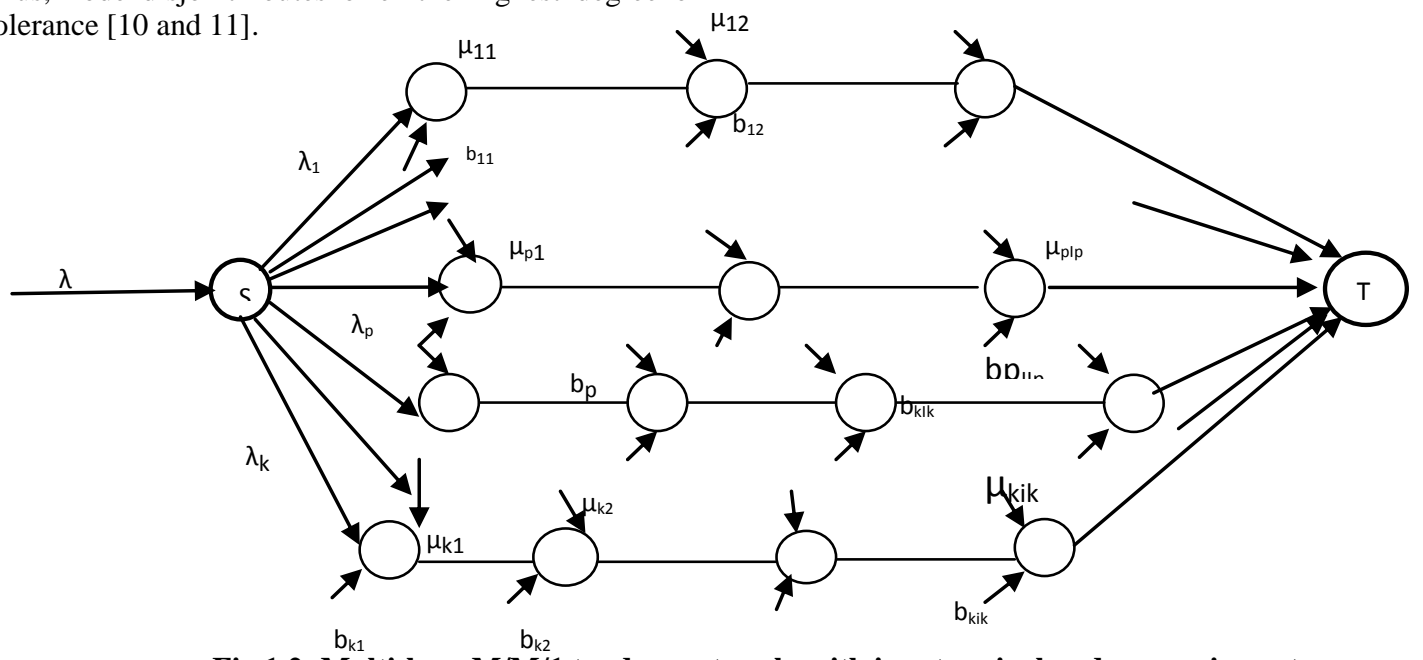

Fig 1.2: Multi-hop, $M / M / 1$ tandem network, with input arrival and processing rate 
Suppose a traffic flow with average passion arrival rate $\lambda$ exist between source and destination, this traffic is then splitted in $\mathrm{k}$-node disjointed paths, the traffic along path $\mathrm{p}$ is $\lambda_{\mathrm{p}},(\mathrm{p}=1,2,-$ $--, \mathrm{k})$.the distribution of traffic $\lambda_{\mathrm{p}},(\mathrm{p}=1,2,--, \mathrm{k})$ is Poisson distributed $[17,18,19$ and 20],with

$$
\sum \lambda_{\mathrm{p}}=\lambda
$$

Let $\mu_{\mathrm{ip}}$ be the average processing rate of the $\mathrm{i}^{\text {th }}$ queue at the $\mathrm{i}^{\text {th }}$ node on path $p$ and $b_{i p}$ is the average arrival of background traffic at the $i^{\text {th }}$ node on path $\mathrm{p}$.

Let us assume that inter path traffic and sub flow traffic are independent and $\mathrm{P}_{\mathrm{i} . \mathrm{p}}$ is the probability that the node of interest remains available during observation.

Although we are studying the problem of adhoc network in static environment ,however it has been realized that node mobility pattern has significant impact on the connectivity graph which influences' the performance of the routing protocols in adhoc network.[21, 22 and 23]

\subsubsection{Single shortest path routing}

In Single path routing entire traffic will be routed along a single shortest path, the mean delay ill be given by

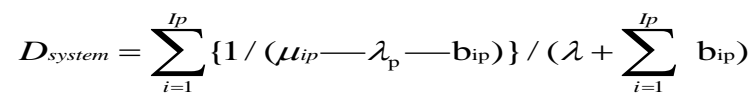

\subsubsection{Splittable $k$ - path routing}

Now if traffic is distributed among $\mathrm{k}$ paths and the distribution of traffic is queuing delay based then of course there is a inverse relation between allocation of traffic and delay for a particular path.

i.e if $\lambda_{\mathrm{p}}$ is traffic allocated to the path $\mathrm{p}$, where $\mathrm{p}=1,2,3, \ldots \ldots \ldots . . \mathrm{k}$, then

$\lambda_{\mathrm{p}} \alpha 1 / \mathrm{D}_{\mathrm{p}}$ Where $\mathrm{D}_{\mathrm{p}}$ is queuing delay for path $\mathrm{p}$.

$\lambda_{\mathrm{p}}=\alpha / \mathrm{D}_{\mathrm{p}}$

$$
\text { and } \sum_{p=1}^{k} \lambda_{p}=\lambda
$$

$$
\begin{gathered}
\sum_{p=1}^{k} \alpha / \mathrm{D}_{\mathrm{p}}=\lambda=\alpha \cdot \sum_{p=1}^{k} 1 / \mathrm{D}_{\mathrm{p}}=\lambda \\
\alpha .\left\{1 / \mathrm{D}_{1}+1 / \mathrm{D}_{2}+1 / \mathrm{D}_{3}+--+1 / \mathrm{D}_{\mathrm{i}}+--+1 / \mathrm{D}_{\mathrm{k}}\right\}=\lambda \\
\alpha=\left\{\left(\lambda \cdot \mathrm{D}_{1} \mathrm{D}_{2 \ldots \ldots . . .} \mathrm{D}_{\mathrm{k}}\right) /\left(\mathrm{D}_{2} \mathrm{D}_{3} \ldots \mathrm{D}_{\mathrm{k}}+--+\mathrm{D}_{1} \mathrm{D}_{2} . . \mathrm{D}_{\mathrm{k}-1}\right)\right\} \\
\quad \text { So } \\
\lambda_{1}=\left\{\left(\lambda \cdot \mathrm{D}_{2} \mathrm{D}_{3} \mathrm{D}_{\mathrm{k}}\right) /\left(\mathrm{D}_{2} \mathrm{D}_{3} \ldots \mathrm{D}_{\mathrm{k}}+--+\mathrm{D}_{1} \mathrm{D}_{2} \ldots \mathrm{D}_{\mathrm{k}-1}\right)\right\}
\end{gathered}
$$

$$
\lambda_{\mathrm{p}}=\left\{\left(\lambda \mathrm{D}_{1} \mathrm{D}_{2} \ldots \mathrm{D}_{\mathrm{p}-1} \mathrm{D}_{\mathrm{p}+1} \ldots \mathrm{D}_{\mathrm{k}}\right) /\left(\mathrm{D}_{2} \mathrm{D}_{3} \ldots \mathrm{D}_{\mathrm{k}}+--+\mathrm{D}_{1} \mathrm{D}_{2} . \mathrm{D}_{\mathrm{k}-1}\right)\right\}
$$

\subsubsection{Mean System Delay}

The back ground traffic depends on node density if nodes in a cell are distributed uniform ally then background traffic may be considered uniform

$$
D_{\text {system }}=\sum_{p=1}^{k} \sum_{i=1}^{I p}\left\{1 /\left(\mu_{i p}-\lambda_{\mathrm{p}}-\mathrm{b}_{\mathrm{ip}}\right)\right\} /\left(\lambda+\sum_{p=1}^{k} \sum_{i=1}^{I p} \mathrm{~b}_{\mathrm{ip}}\right)
$$

Let us consider the case of when background traffic is at most uniform and processing power of each node is $\mu$ then we have $\mathrm{b}_{\mathrm{ip}}=\mathrm{b}, \& \mu_{\mathrm{ip}}=\mu$.

The

$$
D_{\text {system }}=\sum_{p=1}^{k} \sum_{i=1}^{I_{p}}\left\{1 /\left(\mu-\lambda_{\mathrm{p}}-\mathrm{b}\right)\right\} /\left(\lambda+k . I_{p} . \mathrm{b}\right)
$$

\subsubsection{The reliability}

Let us consider $k$ path scenarios, denoting $\mathrm{N}_{1}, \mathrm{~N}_{2},---, \mathrm{N}_{\mathrm{k}}$ as the path lengths of first, second and so on up to k-path respectively .Let $P_{i p}$ is link broken probability of the $i^{\text {th }}$ link in path $p$. The path broken probability $\mathrm{P}_{\mathrm{B}}$ in single path case and k- path case has been analyzed as

A single path route $\mathrm{p}$ is said to be alive until none of the link in the path is broken, if at least one link is broken then the path is broken. Let $\mathrm{P}_{\mathrm{B}, \mathrm{S}}$ denotes the single shortest path broken probability then

$$
\mathrm{P}_{\mathrm{B}, \mathrm{s}}=\left(1-\prod_{i=1}^{N_{1}}\left(1-\mathrm{P}_{\mathrm{ip}}\right)\right)
$$

Let $\mathrm{P}_{\mathrm{B}, \mathrm{k}}$ denotes the path broken probability of k-path model, where all the paths are node disjointed, each path can be considered as a single path carrying a fraction of traffic $\lambda$, and then we have

$$
\mathrm{P}_{\mathrm{B}, \mathrm{k}}=\left\{\left(1-\prod_{i=1}^{N_{1}}\left(1-P_{i 1}\right)\right) .\left(1-\prod_{i=1}^{N 2}\left(1-P_{i 2}\right)\right) \ldots\left(1-\prod_{i=1}^{N k}\left(1-P_{i p}\right)\right)\right\}
$$

For more accurate assessment of breaking probability we also need to consider the influence of mobility and transmission quality.

$$
\left.\mathrm{P}_{\mathrm{B}, \mathrm{k}}=P_{B, S .}\left(1-\prod_{i=1}^{N 2}\left(1-P_{i 2}\right)\right) \ldots .\left(1-\prod_{i=1}^{N k}\left(1-P_{i p}\right)\right)\right\}
$$


$\mathrm{P}_{\mathrm{B}, \mathrm{k}}<\mathrm{P}_{\mathrm{B}, \mathrm{S}}$, , This shows that the reliability of $\mathrm{k}$ - path system is more than single shortest path.

In traffic splitting scheme the traffic par path is low then this will definitely decrease the congestion of the path as congestion factor is $\left(\lambda_{\mathrm{p}} / \mathrm{C}_{\mathrm{ip}}\right)$ where $\mathrm{C}_{\mathrm{ip}}$ is capacity of $\mathrm{i}^{\text {th }}$ link of $\mathrm{p}^{\text {th }}$ path.

\section{Numerical Simulation}

Suppose if node $\mathrm{S}$ (source) has 4 node disjointed routes to the destination $\mathrm{T}$, If $\mathrm{S}$ sends data packets along all three paths, as long as all the routes are not failed, node $\mathrm{T}$ will receive the data and the transmission survives. In single path routing failure of a link/node of the route will stop the entire transmission; thus this approach of distribution of traffic increases the strength of network survival.

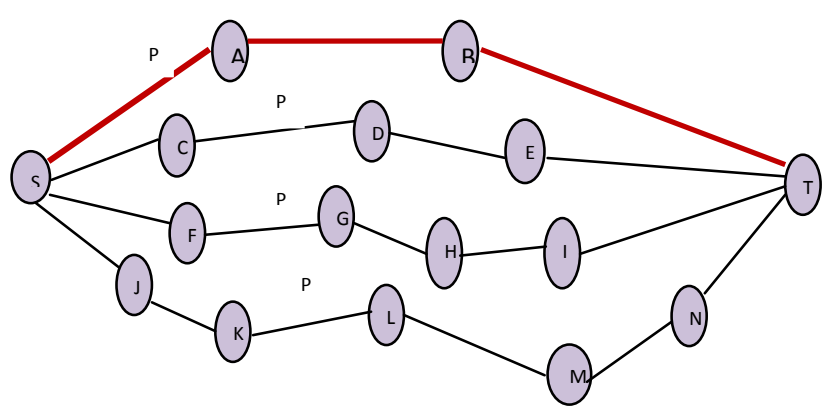

Fig 1.3: Four disjointed routes between source $S$ and destination $\mathbf{T}$

There are 4 disjointed routes between source $\mathrm{S}$ and destination $\mathrm{T}$, out of these paths $\mathrm{P} 1$ is the shortest one containing merely two intermediate node and 3 links, let us suppose the probability of failure of link and nodes are 0.02 and 0.01 respectively, these probabilities are same for all nodes and links in the given network.(Figure-1.3).

Then the probability of failure of path

$$
P_{1}(S-A-B-T), P\left(F P_{1}\right)
$$

is given $b$

$$
P\left(F P_{1}\right)=3 \times 0.02+2 \times 0.01=0.08 \text {. }
$$

If we are applying splittable approach to send the data from $\mathrm{S}$ to $\mathrm{T}$, and using routes $\mathrm{P} 1, \mathrm{P} 2, \mathrm{P} 3$ and $\mathrm{P} 4$ to send data then the probability that all the routes will fail simultaneously can be determined by the relation

$$
\begin{aligned}
& P\left(F P_{1} \cap F P_{2} \cap F P_{3} \cap F P_{4}\right)= \\
& P\left(F P_{1}\right) \times P\left(F P_{2}\right) \times P\left(F P_{3}\right) \times P\left(F P_{4}\right) \\
& P\left(F P_{2}\right)=4 \times 0.02+3 \times 0.01=0.11 \\
& P\left(F P_{3}\right)=5 \times 0.02+4 \times 0.01=0.14 \\
& P\left(F P_{4}\right)=6 \times 0.02+5 \times 0.01=0.17
\end{aligned}
$$

Thus the probability of failure of all the routes is given by

$$
\begin{aligned}
& P\left(F P_{1} \cap F P_{2} \cap F P_{3} \cap F P_{4}\right) \\
= & P\left(F P_{1}\right) \times P\left(F P_{2}\right) \times P\left(F P_{3}\right) \times P\left(F P_{4}\right) \\
& P\left(F P_{1} \cap F P_{2} \cap F P_{3} \cap F P_{4}\right) \\
= & 0.08 \times 0.11 \times 0.14 \times 0.17=0.000209
\end{aligned}
$$

Clearly the $P\left(F P_{1} \cap F P_{2} \cap F P_{3} \cap F P_{4}\right)<P\left(F P_{1}\right)$ thus splittable routing enhances the network reliability and survivability.

K-path splitting $(\mathrm{k}=4)$ : the incoming traffic $\lambda$ is distributed among 4 paths $\mathrm{P} 1, \mathrm{P} 2, \mathrm{P} 3$ and $\mathrm{P} 4$ and the assigned traffic is $\lambda 1$, $\lambda 2, \lambda 3$ and $\lambda 4$ then

$$
\lambda=\lambda_{1}+\lambda_{2}+\lambda_{3}+\lambda_{4}
$$

The queuing delay along path $\mathrm{p}$ is

$$
\begin{aligned}
\mathrm{D}_{\mathrm{p}} & =\sum_{i=1}^{I p}\left\{1 /\left(\mu_{i p}-\mathrm{b}_{\mathrm{ip}}\right)\right\} \\
\mathrm{D}_{\mathrm{p}} & =\sum_{i=1}^{I p}\left\{1 /\left(\mu_{i p}-\mathrm{b}_{\mathrm{ip}}\right)\right\}=\mathrm{I}_{\mathrm{p}} /(\mu-\mathrm{b})
\end{aligned}
$$

$\lambda \in[0,1], \mu=1.0$ unit and $\mathrm{b}=0.10$ unit

For above network queuing delay path wise is

$$
(\mu-\mathrm{b})=0.9
$$

$\mathrm{D} 1=2 / 0.9=2.22, \mathrm{D} 2=3 / 0.9=3.33, \mathrm{D} 3=4 / 0.9=4.44, \mathrm{D} 4=5 / 0.9=5.55$,

For traffic $\quad \lambda=0.8$

$$
\begin{aligned}
& \alpha=\left\{\left(\lambda \cdot \mathrm{D}_{1} \mathrm{D}_{2 \ldots \ldots} \ldots \mathrm{D}_{\mathrm{k}}\right) /\left(\mathrm{D}_{2} \mathrm{D}_{3} \ldots \mathrm{D}_{\mathrm{k}}+-+\mathrm{D}_{1} \mathrm{D}_{2} \ldots \ldots \mathrm{D}_{\mathrm{k}-1}\right)\right\} \\
& \text { so } \quad \alpha=0.691
\end{aligned}
$$


Table 1.1 Distribution of traffic for different values of $\lambda$

\begin{tabular}{|l|l|l|l|l|l|}
\hline $\begin{array}{l}\lambda=0.8 \\
\alpha=0.691\end{array}$ & $\lambda=0.7$ & $\lambda=0.6$ & $\lambda=0.5$ & $\lambda=0.4$ & $\lambda=0.3$ \\
& & $\alpha=0.52$ & $\alpha=0.43$ & $\alpha=0.346$ & $\alpha=0.259$ \\
\hline$\lambda_{1}=0.311$ & $\lambda_{1}=0.272$ & $\lambda_{1}=0.234$ & $\lambda_{1}=0.193$ & $\lambda_{1}=0.150$ & $\lambda_{1}=0.116$ \\
$\lambda_{2}=0.2078$ & $\lambda_{2}=0.182$ & $\lambda_{2}=0.155$ & $\lambda_{2}=0.129$ & $\lambda_{2}=0.103$ & $\lambda_{2}=0.077$ \\
$\lambda_{3}=0.156$ & $\lambda_{3}=0.136$ & $\lambda_{3}=0.117$ & $\lambda_{3}=0.096$ & $\lambda_{3}=0.078$ & $\lambda_{3}=0.0583$ \\
$\lambda_{4}=0.125$ & $\lambda_{4}=0.109$ & $\lambda_{4}=0.093$ & $\lambda_{4}=0.077$ & $\lambda_{4}=0.0623$ & $\lambda_{4}=0.0466$ \\
& & & & & \\
\hline
\end{tabular}

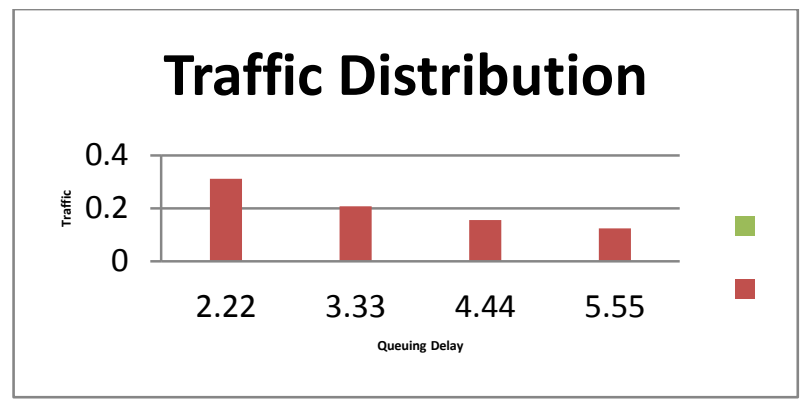

Fig 1.4, for $\lambda=0.80$, Traffic Distribution with respect to Queuing Delay

Mean System Delay (Dsys) (Single path - k-path)

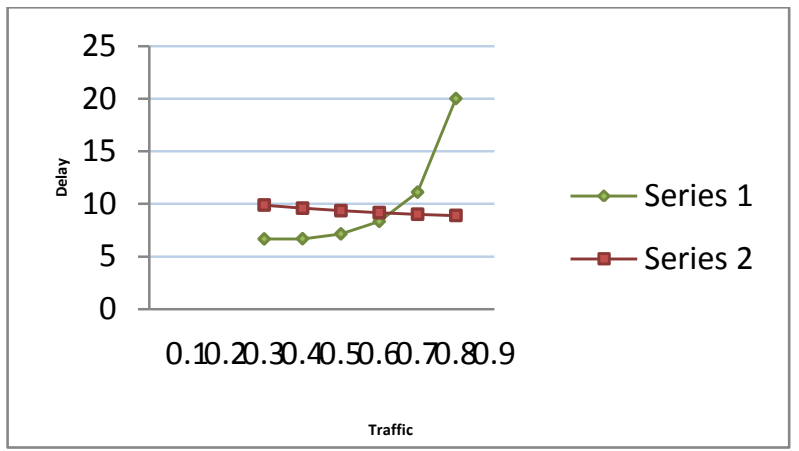

Fig 1.5 Mean System Delay

Table 1.2 Mean system delay

\begin{tabular}{|c|c|c|c|c|c|c|}
\hline $\boldsymbol{\lambda}$ & $\mathbf{0 . 8}$ & $\mathbf{0 . 7}$ & $\mathbf{0 . 6}$ & $\mathbf{0 . 5}$ & $\mathbf{0 . 4}$ & $\mathbf{0 . 3}$ \\
\hline $\begin{array}{c}\text { Dsys } \\
\text { (k- path) }\end{array}$ & 8.89 & 9.00479 & 9.160 & 9.347 & 9.583 & 9.882 \\
\hline $\begin{array}{c}\text { Dsys } \\
\text { (single path) }\end{array}$ & 20 & 11.1 & 8.325 & 7.14 & 6.68 & 6.66 \\
\hline
\end{tabular}

\section{Conclusion}

This paper clearly analyses that splitting of traffic to kdistinct paths provides much reliable transmission system, minimizes the mean system delay and a less congested communication without hot spot in comparison to single shortest path approach. The graph (Figure 1.5)suggest that for less amount of traffic single path routing is better approach but when traffic crosses a particular limit splitting of traffic gives better results. Thus delay based traffic distribution a scheme seems to provide better results in terms of load balancing, minimization of delay and congestion.

\section{Acknowledgement}

We wish to express our sincere gratitude and cordial thanks to Prof. Kamal Ghansala (President Graphic Era University) for his sincere and continual encouragement in preparing this paper; thanks are also due to Dr. R. C. Joshi (Chancellor Graphic Era University) and Dr. Sanjay Jasola (V.C Grahic Era Hill University) for their continual guidance, support and helpful discussion

\section{References}

[1] C.M.d.Carlos and D.P.Agarwal "Adhoc and Sensor Networks", World Scientific Publishing Co. Pte.Ltd. 2006.

[2] D. Bertseakas, R.Gallager, Data Networks, Prentice Hall, 1992.

[3] S.J Lee, M.Gerla, "Split multipath routing with maximally disjointed paths in ad hoc network", in ICC 2001, pp. 867-871, June2001.

[4] M. K.Marina, S. R. Das, "Adhoc on-demand multipath distance path routing", ACM SIGMOBILE mobile computing and communication review, Vol6, No.3, July 2002.

[5] W. Liang, "Minimizing energy and maximizing network life time multicasting in wireless ad hoc network", in IEEE International Conference on Communication, pp. 3375-3380, 2005

[6] G.Chakarbarti, S. Kulkarni, "Load balancing and resource reservation in mobile ad hoc networks ", Ad hoc Networks, pp. 186-203, 2006

[7] P.P. Pham, S. Perreau, "Performance analysis of reactive shortest path and multipath routing mechanism with load balance", in INFOCOM2003, March2003.

[8] Y. Ganjali, A. Keshavarzian, "Load balancing in Adhoc networks, single path routing vs. Multipath routing." INFOCOM2004, March2004.

[9] C. Chen W. W. Z. Li "Multipath Routing Modeling in Ad Hoc Networks" IEEE International Conference on Communications, 2005.

[10] S.J.Lee and M.Gerla "Split multipath routing with maximally disjointed paths in ad hoc network", in ICC 2001,pp867-871,June2001.

[11] Z. QY, V.K.Srikant and Satish KT “A frame work for Reliabile routing in mobile adhoc networks", INFOCOM 2003, pp 270-280.

[12] Qin, F. and Liu, Y. "Multipath routing in mobile adhoc Network", in proceeding of the international symposium on information processing, Huangshan, China, pp. 237 240, 2009

[13] G. I. Iavscu, S. Pierre, A.Quintero ,"QoS routing with traffic distribution in mobile ad hoc networks " In proceeding of ACM journal Computer communication ,Vol.32, No.2, Feburary ,2009.

[14] J.K. Sharma, Operation Research Theory and Applications, MacMillan India limited 2004

[15] D.B. Johnaon and D.A Maltz," Dynamic Source routing in Adhoc wireless Networks", Mobile Computing, Pages 153-181, 1996 
[16] L. Zhang, Z. Zhao, Y. Shu, L. Wang and O.W.W. Yang, "Load Balancing of Multipath Source Routing in Ad Hoc Networks," in ICC 2002, pp. 3197-3201, April 2002.

[17] J.H. Chang, L.Tassiulas, "Maximum lifetime routing in wireless sensor network", Transaction on Networking IEEE/ACM, Vol, 12. No.4. pp. 609-619, 2004.

[18] G.Chakarbarti, S. Kulkarni, “ Load balancing and resource reservation in mobile ad hoc networks ", Ad hoc Networks, pp186-203, 2006

[19] F.Qin and Y.Liu "Multipath routing in mobile adhoc Network", in proceeding of the international symposium on information processing, Huangshan, China,PP 237-240 ,2009

[20] S.C. Dimri, K.C. Purohit, D. Pant, "Improvement of performance of mobile ad hoc network using k-path splittable traffic flow scheme. "International Journal of Computer Technology and Application, Vol. 2 (6), 1911 1917 IJCTA NOV-DEC 2011.

[21] J. N. Kapur, H.C. Saxena, "Mathematical Statistics", S.Chand and Company Ltd. New Delhi, 2007.

[22] L.Wang, L.F. Zhang, Y.T.Shu, M. Dong, and O.W.W. Yang, " Adaptive multipath source routing in wireless Ad hoc networks", IEEE ICC 98, Helsinki, Finland June 2001.

[23]G. I. Iavscu, S. Pierre, A. Quintero ,'QoS routing with traffic distribution in mobile ad hoc networks " In proceeding of ACM journal Computer communication ,Vol.32, No.2, Feburary, 2009. 